\title{
Análise de interação medicamentosa de prescrições médicas contendo antimicrobianos de uma drogaria privada de Minas Gerais
}

\author{
Analysis of the drug interaction of prescriptions containing \\ antimicrobials from a private drugstore in Minas Gerais
Las prescripciones de interacción de fármacos que contienen antimicrobianos en una farmacia privada de Minas Gerais

Patrick Leonardo Nogueira da SILVA ${ }^{(1)}$

Larissa Santos ALVES ${ }^{(2)}$

José Ronivon FONSECA ${ }^{(3)}$

Luçandra Ramos Espírito SANTO ${ }^{(2)}$

Maria Dolores Tiago VAZ ${ }^{(2)}$

${ }^{(1)}$ Fundação Hospitalar do Município de Espinosa - FHUMESP, Espinosa, MG, Brasil.
${ }^{(2)}$ Faculdades Unidas do Norte de Minas - FUNORTE, Montes Claros, MG, Brasil.
${ }^{(3)}$ Universidade Estadual de Montes Claros - UNIMONTES, Montes Claros, MG, Brasil.

Recebido: 20 dez 2016

Revisado: 18 abr 2017

Aceito: 19 maio 2017

Autor de

correspondência:

Patrick Leonardo

Nogueira da Silva

patrick_mocesp70@hotmail.com

Conflito de interesses:

Os autores declaram não

haver nenhum interesse profissional ou pessoal que possa gerar conflito de interesses em relação a este manuscrito.

\begin{abstract}
Resumo
Os antimicrobianos são drogas que têm a capacidade de inibir o crescimento de microrganismos, indicadas, portanto, apenas para o tratamento de infecções microbianas sensíveis. Estas desencadeiam outros sintomas, tais como dor, edema, hipertermia, dentre outros, na qual são controlados com a associação de outros medicamentos. A interação de polifármacos pode proporcionar efeitos colaterais nocivos à saúde do paciente. Este estudo objetivou analisar a interação medicamentosa envolvendo antimicrobianos em prescrições médicas de uma drogaria privada de Minas Gerais. Trata-se de um estudo descritivo, exploratório, observacional, documental, com abordagem quantitativa, na qual foram analisadas 100 prescrições médicas de clientes no período de novembro de 2012. Foi utilizado um formulário como instrumento de coleta de dados. O tratamento dos mesmos se deu por meio de análise estatística uni-variada. Evidenciou-se que a maior parte das prescrições era para mulheres (62\%) na qual se observou que o antibiótico mais prescrito foi a azitromicina (25\%), seguido da amoxicilina (16\%). Essas prescrições continham antiinflamatórios (21\%), porém $24 \%$ pertenciam a outras classes medicamentosas. A terapia antimicrobiana decorreu em um período mínimo de sete dias. Quanto à data entre a prescrição e dispensação dos medicamentos, foi observado um período entre dois a 10 dias. Revelou que $85 \%$ desses clientes eram provenientes de clínicas particulares, enquanto 15\% eram da rede pública. Quanto à via de administração, a mais utilizada foi a via oral (79\%). Portanto, o conhecimento de interações medicamentosas torna-se uma ferramenta, tanto para profissionais dispensadores, quanto por prescritores, na prevenção de possíveis efeitos deletérios que as interações podem causar à saúde.
\end{abstract}

Descritores: Antibacterianos; Infecção; Interações de Medicamentos; Farmácia. 


\begin{abstract}
Antimicrobials are drugs that have the ability to inhibit the growth of microrganisms, thus indicated only for the treatment of sensitive microbial infections. These trigger other symptoms, such as pain, edema, hyperthermia, among others, in which they are controlled with the association of other drugs. The interaction of polyprogesters can provide adverse side effects to the health of the patient. This study aimed to analyze of the drug interaction of prescriptions containing antimicrobials from a private drugstore in Minas Gerais. This is a descriptive, exploratory, observational, documental study with a quantitative approach, in which 100 medical prescriptions of clients in November 2012. A form was used as a collection tool of data. The treatment of these was by means of uni-varied statistical analysis. It was evidenced that most of the prescriptions were for women (62\%) in which the most prescribed antibiotic was azithromycin (25\%), followed by amoxicillin (16\%). These prescriptions contained anti-inflammatories (21\%), but $24 \%$ belonged to other drug classes. The antimicrobial therapy took place in a minimum period of seven days. As for the date between prescription and dispensing of the drugs, a period between two to 10 days was observed. It revealed that $85 \%$ of these clients came from private clinics, while $15 \%$ public network. Regarding the route of administration, the oral route was the most used (79\%). Therefore, the knowledge of drug interactions becomes a tool, both for dispensing professionals and for prescribers, in the prevention of possible deleterious effects that interactions can cause to health.
\end{abstract}

Keywords: Anti-Bacterial Agents; Infection; Drug Interactions; Pharmacy.

\title{
Resumen
}

Los antimicrobianos son fármacos que tienen la capacidad de inhibir el crecimiento de microrganismos enumerados por lo tanto sólo sensibles para el tratamiento de la infección microbiana. Estos desencadenar otros síntomas tales como dolor, hinchazón, hipertermia, entre otros, que son controlados con la combinación de otros medicamentos. La interacción de múltiples fármacos puede proporcionar efectos secundarios perjudiciales para la salud del paciente. Este estudio tuvo como objetivo analizar las prescripciones de interacción de fármacos que contienen antimicrobianos en una farmacia privada de Minas Gerais. Se trata de un estudio descriptivo, exploratorio, documental de observación, con un enfoque cuantitativo, que analizó 100 prescripciones de clientes a partir de noviembre de 2012. Se utilizó una forma como una herramienta de recolección datos. El tratamiento de ellos era a través del análisis estadística univariable. Era evidente que la mayoría de los requisitos era para las mujeres (62\%) en la cual se observó que el antibiótico más comúnmente prescrito es azitromicina (25\%), seguido de la amoxicilina (16\%). Esas prescripciones contienen antiinflamatorios (21\%), pero el $24 \%$ pertenecía a otras clases de fármacos. El tratamiento antimicrobiano se llevó a cabo durante un período mínimo de siete días. A medida que el tiempo entre la prescripción y la dispensación de medicamentos, se observó un período de dos a 10 días. El estudio reveló que el $85 \%$ de los clientes eran de las clínicas privadas, mientras que el $15 \%$ del público. En cuanto a la vía de administración, el más utilizado era oral (79\%). Por lo tanto, el conocimiento de las interacciones farmacológicas se convierte en una herramienta tanto para los dispensadores profesionales, y por los prescriptores, para evitar posibles efectos perjudiciales que las interacciones pueden causar problemas de salud.

Palabras-claves: Antibacterianos; Infección; Interacciones de Drogas; Farmacia. 


\section{Introdução}

ISSN 2179-6750

No início dos anos 40, no século XX, iniciava-se a "era antibiótica", que parecia destinada a vencer a grande batalha contra as infecções, erradicando doenças e aumentado significativamente a expectativa de vida. ${ }^{1}$ Esse surgimento dos antibióticos (ATB) foi uma alternativa, até então segura, de combater microrganismos. ${ }^{2}$

Desde então estes medicamentos são utilizados no tratamento de uma infecção estabelecida ou para impedir o crescimento bacteriano, sem causar danos ao paciente. A ação dos ATB pode ser feita através de alguns mecanismos, dentre eles, atuarem na síntese da parede celular, destruindo as estruturas do microrganismo, como a Penicilina; agir na síntese de proteínas bacterianas, como a eritromicina ou dificultar na síntese de ácidos nucléicos, por exemplo, as Sulfonamidas. ${ }^{2}$

Os ATB são fármacos frequentemente prescritos e quase um terço das prescrições é utilizado incorretamente. A antibioticoterapia apropriada significa não usar ATB na ausência de indicação, nem em esquema errado ou por tempo demasiado. ${ }^{3}$ São vários os fatores que podem influenciar o prescritor quando vai escolher qual ATB utilizar, dentre eles: a condição e o acesso do paciente ao medicamento, as concepções do processo saúde-doença, dentre outros. Ao decidir pela prescrição de um ATB, os prescritores devem preocupar-se com os interesses presentes e futuros dos pacientes e da população como um todo. ${ }^{4,5}$

O uso abusivo de ATB pode acarretar problemas como o desenvolvimento de microrganismos potencialmente resistentes a qualquer tratamento, o que pode levar a um comprometimento no tratamento do paciente e até mesmo levá-lo a óbito, ou eventos adversos que podem levar ao abandono do tratamento medicamentoso e comprometer ainda mais a sensibilidade microbiana. Os efeitos adversos, não só relacionados à ATB, constituem um problema de saúde pública. ${ }^{6}$

Nas últimas décadas, o número de idosos no Brasil cresceu rapidamente, bem como, a preocupação com a saúde desta parcela da população. Neste cenário, a 
institucionalização em casas de repouso aparece como uma alternativa para os cuidados com a saúde dos idosos debilitados ou que vivem sozinhos. Um estudo buscou avaliar a farmacoterapia e a prescrição inadequada para idosos que residem em instituições de longa permanência no sudeste do Brasil. Esta foi realizada em cinco instituições, totalizando uma amostra de 151 indivíduos idosos. O banco de dados foi analisado para identificação de interações medicamentosas, dose diária definida (DDD) e critérios de Beers. Dentre os indivíduos avaliados, 95,36\% consomem algum tipo de medicamento, sendo 3,31 \pm 1,80 medicamentos prescritos por indivíduo. Com base nos critérios de Beers, a prevalência de prescrições inapropriadas foi de $25,83 \%$, sendo também encontradas 70,2\% das prescrições com doses superiores à DDD (Anatomical Therapeutic Chemical [ATC]/World Health Organization [WHO]). Interações medicamentosas potenciais estavam presentes em $54,11 \%$ das prescrições, sendo $81,42 \%$ com grau moderado de gravidade. Os principais medicamentos inapropriados foram prescritos para problemas cardiovasculares e do sistema nervoso. Os idosos institucionalizados apresentam alto consumo e uso inadequado de medicamentos, sendo necessária a intervenção profissional para monitorar as prescrições médicas e melhorar a qualidade do serviço de distribuição de medicamentos a esta parte da população. ${ }^{7}$

Portanto, o presente estudo objetivou analisar a interação medicamentosa envolvendo antimicrobianos em prescrições médicas de uma drogaria privada de Minas Gerais.

\section{Metodologia}

Artigo da monografia intitulada "Análise da interação medicamentosa envolvendo antimicrobianos em drogaria no norte de Minas Gerais" apresentada ao Departamento de Farmácia das Faculdades Unidas do Norte de Minas - FUNORTE. Montes Claros (MG), Brasil. 2012.

Trata-se de um estudo descritivo, exploratório, observacional, documental, com 
abordagem quantitativa, realizada em uma drogaria privada localizada na cidade de Montes Claros, Minas Gerais. O universo da pesquisa apresentou 250 prescrições médicas de usuários de ATB. Estas prescrições são disponibilizadas pelo médico em duas vias na qual a primeira via fica retida na drogaria e a segunda via é devolvida ao cliente. Após aplicação dos critérios de inclusão e exclusão, a amostra foi composta por 100 prescrições médicas recebidas de clientes atendidos na drogaria na qual faziam uso de ATB.

A cidade de Montes Claros localiza-se na região norte do Estado de Minas Gerais. Em 2016, apresentou uma população estimada de 398.288 habitantes, conforme o último censo do Instituto Brasileiro de Geografia e Estatística - IBGE. ${ }^{8}$ Sua extensão territorial compreende uma área de $3.568,941 \mathrm{~km}^{2}$ e sua densidade demográfica equivale a 101,41 hab/ $/ \mathrm{km}^{2}$. Em 2010, estudos afirmam que a população total desta cidade era de 361.915 habitantes. Destes, 8,6\% correspondiam à população idosa na qual é a classe que apresenta maior predisposição de potenciais interações medicamentosas. ${ }^{9}$

De acordo o Departamento de Atenção Básica do Ministério da Saúde - DAB/MS, Montes Claros apresenta 122 equipes de saúde da família implantadas na qual correspondem a uma proporção de $65,9 \%$ da cobertura de saúde da família no município. ${ }^{10}$ Quanto aos planos de saúde, o município de Montes Claros apresenta uma cobertura de $21,5 \%$ de usuários de planos de saúde privado, sendo os principais planos de saúde: UNIMED Norte de Minas, Plano de Saúde Santa Casa, Plano de Saúde São Lucas. ${ }^{11}$

Foram adotados os seguintes critérios de inclusão para participação no estudo: prescrições médicas de usuários de ATB com potenciais interações medicamentosas baseada na classificação ATC; o atendimento e abordagem dos clientes na farmácia ocorreram de forma aleatória, de modo que todas as prescrições com ATB atendidas foram analisadas. Não foram incluídas neste estudo prescrições médicas com rasuras ou ilegíveis.

Utilizou-se um formulário como instrumento de coleta de dados. A mesma ocorreu durante o mês de novembro de 2012. A aplicação do formulário foi feita pela 
pesquisadora do estudo, sendo esta uma acadêmica do curso de graduação em Farmácia das Faculdades Unidas do Norte de Minas - FUNORTE, sob supervisão do farmacêutico responsável pela farmácia, na qual a mesma recebeu o devido treinamento para a realização da pesquisa. Foram analisadas as seguintes variáveis: data entre prescrição e dispensação, gênero, origem das prescrições, via de administração e as interações medicamentosas por meio de dados da literatura científica online estruturada em uma tabela contendo todas as interações já conhecidas.

Os dados foram armazenados por meio do programa StatisticalPackage for the

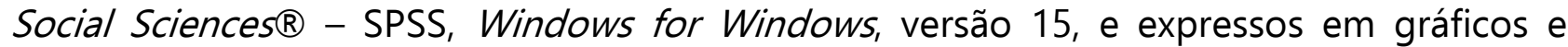
tabelas na qual as mesmas foram construídas por meio do programa Microsoft Exce/ß, Windows for Windows, versão 2010. O tratamento dos dados foi realizado através de análise estatística uni-variada.

Foi encaminhado ao responsável técnico pelo estabelecimento privado um ofício, juntamente com todas as diretrizes do estudo, na qual a sua realização foi autorizada por meio da assinatura do Termo de Concordância Institucional - TCI.

O estudo obedeceu aos preceitos éticos estabelecidos pela Resolução n 466/2012, do Conselho Nacional de Saúde - CNS, na qual regulamenta a realização de pesquisa envolvendo seres humanos. ${ }^{12} \mathrm{O}$ projeto de pesquisa foi apreciado e aprovado pelo Comitê de Ética em Pesquisa das Faculdades Unidas do Norte de Minas - CEP/FUNORTE, sob parecer consubstanciado no 152.931/2012, Certificado de Apresentação para Apreciação Ética - CAAE nº 09159812.0.0000.5141.

Os participantes foram orientados quanto às diretrizes do estudo na qual autorizaram a realização da pesquisa por meio da assinatura do Termo de Consentimento Livre e Esclarecido - TCLE.

\section{Resultados}

Houve prevalência de prescrição para o gênero feminino (62\%) quando comparada 
às prescrições masculinas (38\%), sendo que destas, $5 \%$ são de crianças. Foi revelado que 85\% eram provenientes de clínicas particulares (Tabela 1).

Tabela 1. Perfil socioeconômico dos clientes quanto à prevalência de prescrição dos ATB dispensados na farmácia da rede privada. Montes Claros (MG), 2012.

\begin{tabular}{lll} 
Variáveis & n & $\%$ \\
\hline Gênero & & \\
Feminino & 62 & 62 \\
Masculino & 38 & 38 \\
Proveniência da receita prescrita & & \\
Consultório particular & 85 & 85 \\
Consultório público & 15 & 15 \\
\hline
\end{tabular}

Fonte: Elaborado pelos autores (2012).

Nota: $n=100$.

Em se tratando da frequência de utilização de ATB analisados separadamente por princípio ativo, foi observado que o ATB mais prescrito isoladamente foi a azitromicina (25\%), seguido da amoxicilina (16\%) (Tabela 2).

Tabela 2. Perfil terapêutico dos clientes quanto à prevalência de prescrição dos ATB dispensados na drogaria privada, Montes Claros (MG), 2012.

\begin{tabular}{|c|c|c|c|}
\hline ATB prescritos & $\mathbf{n}$ & $\%$ & Dose/Receita \\
\hline \multicolumn{4}{|l|}{ Uso oral } \\
\hline Azitromicina* & 25 & 25 & 125 \\
\hline Amoxicilina** & 16 & 16 & 448 \\
\hline Amoxicilina + Clavulanato de potássio ${ }^{* *}$ & 10 & 10 & 280 \\
\hline Ciprofloxacino $^{* \star *}$ & 6 & 6 & 84 \\
\hline Levofloxacino $^{* * *}$ & 6 & 6 & 84 \\
\hline Cefalexina $^{* *}$ & 6 & 6 & 168 \\
\hline Sulfametoxazol + Trimetoprima ${ }^{* \star *}$ & 5 & 5 & 70 \\
\hline Ampicilina ${ }^{\star *}$ & 5 & 5 & 140 \\
\hline \multicolumn{4}{|l|}{ Uso tópico } \\
\hline Sulfato de neomicina + Bacitracina $(5 \mathrm{mg}+250 \mathrm{UI})$ & 18 & 18 & 18 tubos \\
\hline \multicolumn{4}{|l|}{ Uso Injetável } \\
\hline Ceftriaxona sódica hemieptaidratada $1 \mathrm{~g}^{\star \star \star \star \star}$ & 3 & 3 & 15 ampolas \\
\hline Total & 79 & 79 & \\
\hline
\end{tabular}

Fonte: Elaborado pelos autores (2012). 
Notas: Posologia prescrita: ${ }^{*} 1$ comprimido ao dia por 5 dias; ${ }^{*} 1$ comprimido de $6 / 6 \mathrm{~h}$ por 7 dias; ${ }^{\star * *} 1$ comprimido de $12 / 12 \mathrm{~h}$ por 7 dias; ${ }^{* \star *} 1$ comprimido ao dia por 14 dias; ${ }^{* \star * *} 1$ ampola ao dia, via intramuscular (IM), por 5 dias; $(n=100)$.

A maior parte das prescrições apresentava duração do tratamento com ATB entre o intervalo de 3-7 dias (51\%), com valores limites de 3 a 28 dias. Quanto à data entre a prescrição e a aquisição do medicamento, foi observado um período entre 2-10 dias. Com relação à via de administração de medicamento, prevaleceu a via oral (79\%) (Tabela 3).

Tabela 3. Perfil clínico dos clientes quanto à prevalência de prescrição dos ATB dispensados na drogaria privada, Montes Claros (MG), 2012.

\begin{tabular}{lcc} 
Variáveis & $\mathbf{n}$ & $\%$ \\
\hline \multicolumn{2}{c}{ Intervalo de duração mínima e máxima do tratamento com ATB } \\
\hline Entre 3-7 dias & 51 & 51 \\
Entre 7-14 dias & 12 & 12 \\
Entre 14-28 dias & 37 & 37 \\
$\quad$ Período entre prescrição e aquisição do medicamento \\
De 2-5 dias & 55 & 55 \\
De 5-8 dias & 35 & 35 \\
De 8-10 dias & 10 & 10 \\
$\quad$ Via de administração de medicamento mais prescrita \\
Oral & 79 & 79 \\
Tópica & 18 & 18 \\
Intramuscular & 3 & 3 \\
laboração própria (2012). & &
\end{tabular}

Os resultados demonstraram que $21 \%$ das prescrições simultâneas de ATB foram com antiinflamatórios na qual não apresentou manifestação desfavorável; 19\% com analgésicos (fármacos utilizados para o alívio da dor) na qual não apresentou manifestação desfavorável com ATB; 19\% com corticóides; e 17\% com antialérgicos (Gráfico 1).

Salienta-se que foram encontradas outras classes de medicamento de igual importância associadas às prescrições com ATB, de modo a compor a categoria "outros" 
do Gráfico 1, sendo estas: anti-hipertensivos ( $n=10 ; 41,6 \%)$, hipoglicêmicos $(n=3 ; 12,5 \%)$, antiespasmódicos $(n=1 ; 4,3 \%)$ e antitérmicos $(n=10 ; 41,6 \%)$, totalizando um total de 24 prescrições.

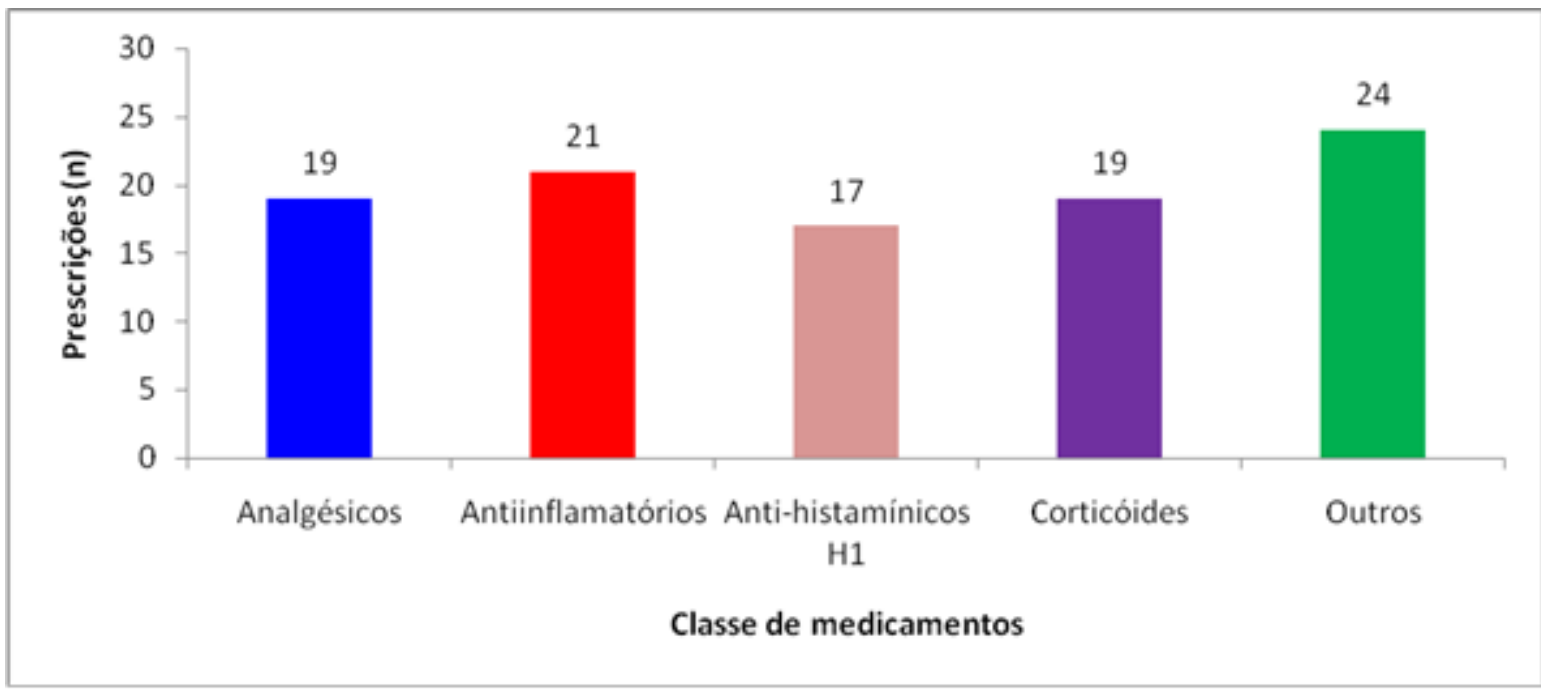

Gráfico 1. Prevalência de prescrição simultânea dos ATB com outras classes de medicamentos, Montes Claros (MG), 2012.

O Quadro 1 apresenta as interações já conhecidas entre uma parte de antibióticos com outras classes medicamentosas, de modo a complementar as informações estabelecidas pelo Gráfico 1.

Quadro 1. Relação de possíveis interações medicamentosas segundo seu efeito, Montes Claros (MG), 2012.

\begin{tabular}{|c|c|c|}
\hline Medicamento & ATB & Efeito \\
\hline Metotrexato & \multirow{4}{*}{$\begin{array}{l}\text { AMOXICILINA + } \\
\text { CLAVULANATO } \\
\text { DE POTÁSSIO }\end{array}$} & $\begin{array}{l}\text { Aumenta os efeitos tóxicos do } \\
\text { metotrexato. }\end{array}$ \\
\hline Venlafaxina & & $\begin{array}{l}\text { Aumenta o risco da síndrome } \\
\text { serotoninérgica. }\end{array}$ \\
\hline Contraceptivos orais & & $\begin{array}{l}\text { Diminui a eficácia dos } \\
\text { contraceptivos. }\end{array}$ \\
\hline Warfarina & & Aumenta o risco de sangramento. \\
\hline Contraceptivos orais & \multirow{2}{*}{ AMPICILINA } & $\begin{array}{l}\text { Diminui a eficácia dos } \\
\text { contraceptivos. }\end{array}$ \\
\hline Entacapone & & $\begin{array}{l}\text { Aumenta os efeitos adversos do } \\
\text { entacapone (diarréia e discinesia). }\end{array}$ \\
\hline
\end{tabular}

9 https://doi.org/10.14295/jmphc.v10i0.481 


\begin{tabular}{|c|c|c|}
\hline $\begin{array}{l}\text { Inibidores da bomba de prótons } \\
\text { (omeprazol, lanzoprazol etc.) }\end{array}$ & & Diminui os efeitos da ampicilina. \\
\hline Colestiramina & \multirow[b]{2}{*}{ CEFALEXINA } & Diminui a eficácia da cefalexina. \\
\hline Metformina & & $\begin{array}{l}\text { Aumento dos efeitos } \\
\text { hipoglicemiantes. }\end{array}$ \\
\hline $\begin{array}{l}\text { Substância que contenha cálcio } \\
\text { (gluconato de cálcio, fosfato de } \\
\text { cálcio, ringer lactato, ringer simples) }\end{array}$ & \multirow{2}{*}{ CEFTRIAXONA } & $\begin{array}{l}\text { Risco de morte por eventos } \\
\text { cardiopulmonares. }\end{array}$ \\
\hline Ciclosporina & & $\begin{array}{l}\text { Aumenta a toxicidade da } \\
\text { ciclosporina (disfunção renal, } \\
\text { parestesias e colestase). }\end{array}$ \\
\hline Tizanidina & \multirow{10}{*}{ CIPROFLOXACINA } & Hipotensão e efeito sedativo. \\
\hline Amiodarona & & $\begin{array}{l}\text { Aumenta os riscos de } \\
\text { cardiotoxicidade, causando arritmia } \\
\text { cardíaca severa, taquicardia } \\
\text { ventricular (TV) e fibrilação. }\end{array}$ \\
\hline $\begin{array}{l}\text { Antiarrítmicos de classe I (quinidina, } \\
\text { procainamida, disopiramida) }\end{array}$ & & $\begin{array}{l}\text { Aumenta os riscos de } \\
\text { cardiotoxicidade, causando arritmia } \\
\text { cardíaca severa, TV e fibrilação. }\end{array}$ \\
\hline $\begin{array}{l}\text { Hipoglicemiantes orais (acarbose, } \\
\text { clorporpamida, glipizida) }\end{array}$ & & $\begin{array}{l}\text { Causa hipoglicemia e às vezes } \\
\text { hiperglicemia. }\end{array}$ \\
\hline Insulina & & $\begin{array}{l}\text { Causa hipoglicemia e às vezes } \\
\text { hiperglicemia. }\end{array}$ \\
\hline Sinvastatina & & $\begin{array}{l}\text { Aumenta o risco de miopatia e } \\
\text { rabdomiólise. }\end{array}$ \\
\hline Sotalol & & $\begin{array}{l}\text { Aumenta os riscos de } \\
\text { cardiotoxicidade, causando arritmia } \\
\text { cardíaca severa, TV e fibrilação. }\end{array}$ \\
\hline Sucralfoto & & $\begin{array}{l}\text { Diminuir a eficácia da ciprofloxacina } \\
\text { quando administrado os dois } \\
\text { medicamentos por via oral. }\end{array}$ \\
\hline Teofilina & & $\begin{array}{l}\text { Aumenta o risco de toxicidade da } \\
\text { teofilina (náuseas, vômitos, } \\
\text { palpitações e convulsões). }\end{array}$ \\
\hline Antiácidos & & $\begin{array}{l}\text { Diminuição da eficácia da } \\
\text { ciprofloxacina quando administrado } \\
\text { os dois medicamentos por via oral. }\end{array}$ \\
\hline
\end{tabular}




\begin{tabular}{|c|c|c|}
\hline Cálcio & & $\begin{array}{l}\text { Diminuição da eficácia da } \\
\text { ciprofloxacina quando administrado } \\
\text { os dois medicamentos por via oral. }\end{array}$ \\
\hline $\begin{array}{l}\text { Corticóide (dexametasona, } \\
\text { betametasona, prednisona etc.) }\end{array}$ & & $\begin{array}{l}\text { Aumenta o risco de ruptura de } \\
\text { tendão. }\end{array}$ \\
\hline Cloroquina & & $\begin{array}{l}\text { Diminuição da eficácia da } \\
\text { ciprofloxacina quando administrado } \\
\text { os dois medicamentos por via oral. }\end{array}$ \\
\hline Diclofenaco & & $\begin{array}{l}\text { Aumenta a toxicidade da } \\
\text { ciprofloxacina (nervosismo, náuseas, } \\
\text { diarréia). }\end{array}$ \\
\hline Fenitoína & & $\begin{array}{l}\text { Aumenta ou diminui os níveis de } \\
\text { fenitoína. }\end{array}$ \\
\hline Sulfato ferroso & & $\begin{array}{l}\text { Diminuição da eficácia da } \\
\text { ciprofloxacina quando administrado } \\
\text { os dois medicamentos por via oral. }\end{array}$ \\
\hline Eritromicina & & $\left.2^{\circ}\right)$ Efeitos antagônicos. \\
\hline $\begin{array}{l}\text { Bloqueadores neuromusculares } \\
\text { (pancurônio, atracúrio etc.) }\end{array}$ & CLINDAMICINA & $\begin{array}{l}\text { Potencializa os efeitos dos } \\
\text { bloqueadores musculares podendo } \\
\text { causa depressão respiratória. }\end{array}$ \\
\hline
\end{tabular}

Fonte: Elaborado pelos autores (2012).

A Tabela 5 complementa as informações do Gráfico 1 de modo a informar a relação municipal de medicamentos por classificação terapêutica via sistema ATC. Estes fármacos contidos na Tabela 5 são os mais prescritos pelos médicos, a depender do diagnóstico, bem como do tipo específico do seu tratamento.

Tabela 5. Relação municipal de medicamentos por classificação terapêutica via sistema ATC. Montes Claros (MG), 2012.

\begin{tabular}{|c|c|c|}
\hline & $\begin{array}{l}\text { Denominação } \\
\text { genérica }\end{array}$ & $\begin{array}{l}\text { DDD } \\
\text { (mg) }\end{array}$ \\
\hline \multicolumn{3}{|c|}{ A03D - Antiespasmódicos associados com analgésicos } \\
\hline A03DB04 & $\begin{array}{l}\text { Butilbrometo de } \\
\text { escopolamina + } \\
\text { dipirona }\end{array}$ & \\
\hline \multicolumn{3}{|c|}{ A10B - Hipoglicemiantes orais } \\
\hline
\end{tabular}


A10BA02

A10BA02

A10BB01

A10BB09
Metformina

comprimido $500 \mathrm{mg}$

Metformina

comprimido $850 \mathrm{mg}$

2000

Glibenclamida

comprimido $5 \mathrm{mg}$

10

Gliclazida

comprimido de 160

liberação lenta $30 \mathrm{mg}$

C02A - Agentes antiadrenérgicos de ação central

C02AB01

Metildopa
comprimido $500 \mathrm{mg}$

C02C - Antagonistas alfa-adrenérgico

$\begin{array}{ll}\mathrm{C} 02 \mathrm{CA} 04 & \text { Doxazosina } \\ & \text { comprimido } 4 \mathrm{mg}\end{array}$

4

C02D - Vasodilatadores diretos - agentes que atuam no músculo liso das artérias

\begin{tabular}{|c|c|c|}
\hline C02DB02 & $\begin{array}{l}\text { Hidralazinadrágea } \\
25 \mathrm{mg}\end{array}$ & 100 \\
\hline C02DB02 & $\begin{array}{l}\text { Hidralazina drágea } \\
50 \mathrm{mg}\end{array}$ & 100 \\
\hline C02DB02 & $\begin{array}{l}\text { Hidralazina solução } \\
\text { injetável } 20 \mathrm{mg} / \mathrm{mL}\end{array}$ & \\
\hline C02DD01 & $\begin{array}{l}\text { Nitruprussiato de } \\
\text { sódio solução } \\
\text { injetável } 25 \mathrm{mg} / \mathrm{mL}\end{array}$ & 50 \\
\hline \multicolumn{3}{|c|}{ C03A - Diuréticos tiazídicos } \\
\hline С03АA03 & $\begin{array}{l}\text { Hidroclorotiazida } \\
\text { comprimido } 25 \mathrm{mg}\end{array}$ & 25 \\
\hline \multicolumn{3}{|c|}{ C03C - Diuréticos de alça } \\
\hline C03CA01 & $\begin{array}{l}\text { Furosemida } \\
\text { comprimido } 40 \mathrm{mg}\end{array}$ & 40 \\
\hline C03CA01 & $\begin{array}{l}\text { Furosemida solução } \\
\text { injetável } 10 \mathrm{mg} / \mathrm{mL}\end{array}$ & 40 \\
\hline \multicolumn{3}{|c|}{ C03DA - Antagonista de aldosterona } \\
\hline C03DA01 & $\begin{array}{l}\text { Espironolactona } \\
\text { comprimido } 25 \mathrm{mg}\end{array}$ & 75 \\
\hline
\end{tabular}


C03DA01

Espironolactona comprimido $100 \mathrm{mg}$

C07AA - Agentes beta-bloqueadores, não seletivos

\begin{tabular}{|c|c|c|}
\hline C07AA05 & $\begin{array}{l}\text { Propranolol } \\
\text { comprimido } 40 \mathrm{mg}\end{array}$ & 160 \\
\hline \multicolumn{3}{|c|}{ C07AB - Agentes beta-bloqueadores, seletivos } \\
\hline C07AB03 & $\begin{array}{l}\text { Atenolol comprimido } \\
50 \mathrm{mg}\end{array}$ & 75 \\
\hline C07AB03 & $\begin{array}{l}\text { Atenolol comprimido } \\
100 \mathrm{mg}\end{array}$ & 75 \\
\hline \multicolumn{3}{|c|}{ C07AG - Agentes alfa e beta-bloqueadores } \\
\hline C07AG02 & $\begin{array}{l}\text { Carvedilol } \\
\text { comprimido } 12,5 \mathrm{mg}\end{array}$ & 37,5 \\
\hline C07AG02 & $\begin{array}{l}\text { Carvedilol } \\
\text { comprimido } 6,25 \mathrm{mg}\end{array}$ & 37,5 \\
\hline C07AG02 & $\begin{array}{l}\text { Carvedilol } \\
\text { comprimido 25mg }\end{array}$ & 37,5 \\
\hline C07AG02 & $\begin{array}{l}\text { Carvedilol } \\
\text { comprimido } 3,125 \mathrm{mg}\end{array}$ & 37,5 \\
\hline \multicolumn{3}{|c|}{ C09A - Inibidores da ECA } \\
\hline C09AA01 & $\begin{array}{l}\text { Captopril comprimido } \\
25 \mathrm{mg}\end{array}$ & 50 \\
\hline C09AA02 & $\begin{array}{l}\text { Enalapril comprimido } \\
20 \mathrm{mg}\end{array}$ & 10 \\
\hline C09AA02 & $\begin{array}{l}\text { Enalapril comprimido } \\
10 \mathrm{mg}\end{array}$ & 10 \\
\hline C09AA02 & $\begin{array}{l}\text { Enalapril comprimido } \\
5 \mathrm{mg}\end{array}$ & 10 \\
\hline \multicolumn{3}{|c|}{ C09CA - Antagonista de angiotensina II } \\
\hline C09CA01 & $\begin{array}{l}\text { Losartana } \\
\text { comprimido } 25 \mathrm{mg}\end{array}$ & 50 \\
\hline C09CA01 & $\begin{array}{l}\text { Losartana } \\
\text { comprimido 50mg }\end{array}$ & 50 \\
\hline
\end{tabular}

D07 - Corticosteróides, preparação dermatológica

D07AA02

D07AB19
Hidrocortisona creme

$10 \mathrm{mg} / \mathrm{g}$

Dexametasona creme

$0,1 \%$ 
H02AB01

H02AB02

$\mathrm{H} 02 \mathrm{AB} 02$

H02AB04

H02AB06

H02AB07

H02AB07

H02AB09

H02AB09
Betametasona,

fosfato solução

injetável $4 \mathrm{mg} / \mathrm{mL}$

Dexametasona

comprimido $4 \mathrm{mg}$

Dexametasona

solução injetável

$4 \mathrm{mg} / \mathrm{mL}$

Metilprednisolona

solução injetável

500mg

Prednisolona, fosfato

sódico solução oral

$1,34 \mathrm{mg} / \mathrm{mL}$ (eq.

$1 \mathrm{mg} / \mathrm{mL}$ de

prednisolona)

Prednisona

comprimido $20 \mathrm{mg}$

Prednisona

comprimido $5 \mathrm{mg}$

Hidrocortisona

solução injetável

30

100mg

Hidrocortisona

solução injetável

$500 \mathrm{mg}$

M01A - Agentes antiinflamatórios e antirreumáticos não-esteróides

M01AB05

Diclofenaco de sódio

solução injetável

100

$25 \mathrm{mg} / \mathrm{mL}$

Ibuprofeno

M01AE01

comprimido $600 \mathrm{mg}$

1200

Ibuprofeno

M01AE01

suspensao oral

$50 \mathrm{mg} / \mathrm{mL}$

Ibuprofeno

M01AE01

N02B - Outros analgésicos e antipiréticos 
N02BB02

N02BB52

N02BE01

N02BE01

N02BE51

N02BE51
N02BB02

N02BB02

Dipirona solução oral

$500 \mathrm{mg} / \mathrm{mL}$

Dipirona solução injetável $500 \mathrm{mg} / \mathrm{mL}$

Dipirona comprimido $500 \mathrm{mg}$

Dipirona +

prometazina + adifenina solução injetável $750 \mathrm{mg}+$ $25 \mathrm{mg}+25 \mathrm{mg}$

Paracetamol comprimido $500 \mathrm{mg}$ Paracetamol solução oral $200 \mathrm{mg} / \mathrm{mL}$

Paracetamol + codeína (comprimido) $500 \mathrm{mg}+30 \mathrm{mg}$

Paracetamol + codeína (cp) 500 mg $+7,5 \mathrm{mg}$

R06 - Anti-histamínicos de uso sistêmicos

R06AB02

R06AB02

R06AD02

R06AD02

R06AX13

R06AX13
Dexclorfeniramina comprimido $2 \mathrm{mg}$

Dexclorfeniramina solução oral $0,4 \%$ $\mathrm{mg} / \mathrm{mL}$

Prometazina comprimido $25 \mathrm{mg}$

Prometazina solução injetável $25 \mathrm{mg} / \mathrm{mL}$

Loratadina comprimido $10 \mathrm{mg}$

Loratadina suspensão oral $1 \mathrm{mg} / \mathrm{mL}$
3000

3000

3000

3000

3000

Fonte: Elaborado pelos autores (2012).

\section{Discussão}

A dispensação de ATB deve obedecer a normas exigidas pela Agência Nacional de Vigilância Sanitária - ANVISA. Essas normas estão esclarecidas na Resolução da Diretoria 
Colegiada - RDC n²0, de 05 de maio de 2011, que determina prescrições em receituário simples de duas vias, contendo nome completo, idade e sexo do paciente, e tendo este validade de dez dias. A maior parte dos ATB apresenta um período profilático mínimo de sete dias na qual pode ser ampliado por mais sete ou quatorze dias a depender do tipo e grau da infecção. ${ }^{13}$ De acordo com a ANVISA, esse informe técnico é uma maneira de profissionais da área da saúde salientarem quanto à dispensação e controle de ATB, visando estabelecer qualidade e segurança para farmacêuticos e usuários. Neste estudo, a maior parte dos pacientes fez o tratamento por no máximo sete dias na qual a medicação foi adquirida com tempo inferior a 10 dias pós-prescrição corroborando com o preconizado.

Em um estudo foi relatado que mulheres apresentam postura diferente em relação às doenças e ao conceito de saúde, sendo mais inclinadas a prestar atenção aos sinais e sintomas e procurar assistência mais frequentemente que os homens. ${ }^{14}$ Consequentemente, o gênero pode influenciar o consumo de medicamentos, sendo as mulheres as mais prováveis usuárias. Na drogaria pesquisada, as mulheres apresentaram maior prevalência nas prescrições, sendo estas, em sua maior parte, advindas de clínicas particulares. Infere-se que o sistema público apresenta fragilidades quanto ao atendimento de toda a demanda populacional de modo a não disponibilizar vagas para todos e os mesmos terem que fazer uso do sistema privado. Cabe ressaltar que uma mínima parte da amostra (5\%) era composta por crianças, de modo a aumentar o risco de um efeito adverso em decorrência da interação medicamentosa.

A via oral é considerada a mais segura, conveniente e econômica. Corroborando com estes resultados, um estudioso observou em sua pesquisa que o benefício real da via oral está condicionada a fatores como efetividade terapêutica, tolerância gastrointestinal, incidência de eventos adversos, perfil de segurança com risco mínimo de toxicidade e números de tomadas diárias necessário. ${ }^{15} \mathrm{~A}$ azitromicina é considerada como uma boa opção para alguns tratamentos, visto que este fármaco apresenta características favoráveis 
a sua administração dentre elas, amplo espectro de ação, baixa incidência de efeitos colaterais e significativa penetração tecidual, o que contribui para melhor absorção do medicamento. ${ }^{16}$ Em outro estudo, observou-se que a amoxicilina, por ser um ATB de amplo espectro, é considerado como a melhor escolha para tratamentos de alguns tipos de infecções, é uma penicilina semissintética do tipo $\beta$-lactamase que possui espectro de atividade antibacteriana superior a de outras penicilinas. ${ }^{17}$

Quanto à prescrição de amoxicilina associado ao clavulanato, a combinação do ácido clavulânico com a amoxicilina é o exemplo de maior sucesso do uso de ATB $\beta$ lactâmico sensível à $\beta$-lactamase, juntamente com um inibidor desta enzima. ${ }^{18}$ Esta combinação é utilizada no combate a diferentes enfermidades com grande eficácia se comparada a outros medicamentos como, por exemplo, no tratamento de infecções do trato respiratório com resposta positiva superior se comparado à azitromicina, cefixima ou ciprofloxacina nos casos de bronquite crônica. ${ }^{19}$ A amoxicilina associada ao clavulanato pode causar alguns efeitos adversos, como aumento dos efeitos tóxicos de alguns fármacos, aumento da síndrome serotoninérgica, diminui a eficácia dos contraceptivos e aumento dos riscos de sangramento. ${ }^{3}$

As interações envolvendo o ciprofloxacino com outras substancias, sendo algumas destas a tizanidina, amiodarona, antiarrítmicos de classe I (quinidina, procainamida, disopiramida), hipoglicemiantes orais (acarbose, clopropamida, glimeperida, glipizida), insulina e sinvastatina, pode causar hipotensão e efeito sedativo, aumento do risco de cardiotoxicidade, hipoglicemia, miopatia, aumento do risco de ruptura do tendão. ${ }^{19}$ Já o levofloxacino, pode contribuir com efeitos negativos quando combinados com alguns medicamentos, sendo alguns destes a tizanidina, amiodarona, antiarrítmicos de classe I (quinidina, procainamida, disopiramida), clorpromazina, insulina e warfarina, e esses efeitos podem ser hipotensão, cardiotoxicidade, hipoglicemia e sangramento. É importante e necessário salientar quanto à importância das interações medicamentosas, pois essas interações podem ou não contribuir de acordo com os fatores de risco de cada paciente. 
Algumas pessoas são mais propensas às interações medicamentosas como, por exemplo, pacientes idosos, pós-cirúrgicos e imunodeprimidos. A relação entre a dose máxima tolerada e a dose terapêutica, equivalente ao índice terapêutico, é o que estabelece o limite para o bem-estar de cada paciente. ${ }^{20}$

As interações medicamentosas podem ser prejudiciais não só pelo fato de alguns pacientes terem ou não fatores de risco, mas também é passível de ocorrer ações negativas com relação ao maior número de medicamentos utilizados por uma mesma pessoa. Além disso, a eficácia do medicamento pode diminuir, isso faz com que a quantidade de medicamentos ingeridos aumente, ocasionando o surgimento de outras enfermidades. $^{21}$ A politerapia é uma prática comum na medicina, pois, para muitas enfermidades e para muitos médicos, essa prática ajuda no aumento da eficácia do tratamento, mas em contrapartida, esse pode causar um efeito contrário, visto que 10-15\% de casos de interações medicamentosas causam toxicidade ou a perda da eficácia do medicamento. 22

Um estudo mostrou que $3,8 \%$ das pessoas que dão entrada nos hospitais são por causa de problemas relacionados às interações medicamentosas. ${ }^{23}$ Os ATB são os medicamentos mais prescritos em hospitais, as interações envolvendo ATB, em alguns casos, podem ser de grande risco para a saúde do paciente, pois as chances do microrganismo se tornar mais resistente quando associado a outros medicamentos se torna maior e, além disso, pode proporcionar um maior custo para o usuário.

Quanto maior as interações, maior podem ser os danos à saúde, pois as interações envolvendo o uso simultâneo de antiinflamatórios e ATB, tal como o diclofenaco e o ciprofloxacino, podem gerar grandes riscos para o paciente causando problemas no sistema nervoso central - SNC e também convulsões. ${ }^{24}$ Alguns antiinflamatórios combinados com outros medicamentos podem provocar reações adversas como irritação, úlcera gástrica, causando hemorragia, insuficiência renal. ${ }^{21}$ É imprescindível que os profissionais da saúde estejam atentos às prescrições médicas, principalmente quando se 
trata de crianças e idosos, já que a vulnerabilidade destes são maiores do que em outros pacientes, optando sempre em realizar um trabalho que possa oferecer qualidade e seguranças a seus usuários.

O uso de antialérgicos concomitante a alguns ATB podem causar redução da eficácia do medicamento. Pode-se ter um aumento nas concentrações do anti-H1 (antagonistas dos receptores $\mathrm{H} 1$ ), aumentando a sua biodisponibilidade $\mathrm{e}$ assim intensificando seus efeitos adversos, tal como acontece quando se administra drogas que inibem competitivamente os seus metabólitos pelo CYP. ${ }^{25}$ A associação entre ATB e corticóides podem desencadear mucormicoses, formar orbito-ripo-cerebral, pulmonar e gastrintestinal havendo complicações caso o paciente seja portador de diabetes mellitus (DM) descompensado, em cetoacidose. ${ }^{26}$

\section{Conclusão}

Pode-se observar neste estudo que, na dispensação dos medicamentos, todas as normas foram seguidas de acordo com as instruções da ANVISA, contribuindo, assim, com o custo-benefício dos pacientes. É notório observar que, quanto maior o número de fármacos prescritos, maior a probabilidade de potenciais interações medicamentosas, com aumento de riscos para pacientes.

As mulheres apresentam maior predisposição aos cuidados com a saúde quando comparadas aos homens, tendo em vista que o maior número de prescrições é de pacientes do gênero feminino. O ATB oral mais prescrito foi a azitromicina para o tratamento das infecções. Não foram encontrados em estudos científicos quaisquer efeitos colaterais advindos da interação deste com outras classes medicamentosas, podendo inferir que o mesmo apresenta um padrão de segurança estável ao uso.

A maior parte das infecções, bem como os sinais e sintomas secundários propiciados pelas mesmas, regrediam em um intervalo de tempo mínimo para o tratamento (3-7 dias), de modo a constatar a eficácia do seu uso sem a manifestação 
relatada pelos usuários de sintomas desenvolvidos pela interação dos medicamentos.

Dessa forma, pode-se concluir que as possíveis e potenciais interações medicamentosas são muito frequentes entre os ATB e, principalmente, antiinflamatórios, que são medicamentos comumente associado aos tratamentos. Portanto, o conhecimento das interações medicamentosas torna-se uma ferramenta, tanto para profissionais dispensadores, quanto por prescritores, na prevenção de possíveis efeitos deletérios que as interações podem causar à saúde.

\section{Referências}

1. França FB, Costa AC. Perfil farmacoterapêutico de pacientes em uso de antimicrobianos em hospital privado, em Fortaleza - CE. Rev Bras Prom Saude. 2006;19(4):224-8. http://dx.doi.org/10.5020/18061230.2006.p224.

2. Nicolini $\mathrm{P}$, Nascimento JWL, Greco KV, Menezes FG. Fatores relacionados à prescrição médica de antibióticos em farmácia pública da região Oeste da cidade de São Paulo. Cienc Saude Colet. 2008;13(Supl 1):689-96. http://dx.doi.org/10.1590/S1413-81232008000700018.

3. Oliveira KR, Destefani SRA. Perfil da prescrição e dispensação de antibióticos para crianças em uma Unidade Básica de Saúde (UBS) no município de Ijuí - RS. Rev Cienc Farm Básica Apl. 2011;32(3):395-401.

4. Svetitsky S, Leibovici L, Paul M. Comparative efficacy and safety of vancomycin versus teicoplanin: systematic review and meta-analysis. Antimicrob Agents Chemother. 2009;53(10):4069-79. http://dx.doi.org/10.1128/AAC.00341-09.

5. Santos $V$, Nitrini SMOO. Indicadores do uso de medicamentos prescritos e de assistência ao paciente de serviços de saúde. Rev Saude Publica. 2004;38(6):818-26. http://dx.doi.org/10.1590/S0034-89102004000600010.

6. Louro E, Romano-Lieber NS, Ribeiro E. Eventos adversos a antibióticos em pacientes internados em um hospital universitário. Rev Saude Publica. 2007;41(6):1042-8. http://dx.doi.org/10.1590/S0034-89102006005000049. 
7. Pinto MCX, Malaquias DP, Ferré F, Pinheiro MLP. Potentially inappropriate medication use among institutionalized elderly individuals in southeastern Brazil. Braz J Pharm Sci. 2013;49(4):709-17. http://dx.doi.org/10.1590/S1984-82502013000400010.

8. Instituto Brasileiro de Geografia e Estatística. Censo demográfico 2016: Montes Claros e Minas Gerais. Rio de Janeiro: IBGE; 2016.

9. Araújo DD, Azevedo RS, Chianca TCM. Perfil demográfico da população idosa de Montes Claros, Minas Gerais e Brasil. Rev Enferm Cent O Min. 2011;1(4):462-9. http://dx.doi.org/10.19175/recom.v0i0.151.

10. Brasil. Ministério da Saúde. Secretaria de Atenção à Saúde. Departamento de Atenção Básica. Teto, credenciamento e implantação das estratégias de agentes comunitários de saúde, saúde da família e saúde bucal. Brasília, DF: MS; 2017.

11. Brasil. Ministério da Saúde. Rede Interagencial de Informações para a Saúde. Departamento de Informática do Sistema Único de Saúde. Proporção da população coberta por planos privados de saúde - ANS. Brasília, DF): MS; 2012.

12. Brasil. Ministério da Saúde. Conselho Nacional de Saúde. Resolução nº 466, de 12 de dezembro de 2012. Aprova as diretrizes e normas regulamentadoras de pesquisas envolvendo seres humanos. Brasília, DF: MS; 2012.

13. Brasil. Ministério da Saúde. Agência Nacional de Vigilância Sanitária. Resolução da Diretoria Colegiada $(\mathrm{RDC}) \mathrm{n}^{\circ}$ 20, de 05 de maio de 2011. Dispõe sobre o controle de medicamentos à base de substâncias classificadas como antimicrobianos, de uso sob prescrição, isolado ou em associação. Brasília, DF: MS; 2011.

14. Flores LM, Mengue SS. Uso de medicamentos por idosos em região do sul do Brasil. Rev Saude Publica. 2005;39(6):924-9. http://dx.doi.org/10.1590/S0034-89102005000600009.

15. Wynn RL, Cook J. Does ibuprofen block the cardioprotective effects of aspirin in dental patients? Gen Dent. 2006;54(1):6-9. 
16. Bergamaschi CC, Montan MF, Cogo K, Franco GCN, Groppo FC, Volpato MC, et al. Interações medicamentosas: analgésicos, anti-inflamatórios e antibióticos (parte II). Rev Cir Traumatol Buco-Maxilo-Fac. 2007;7(2):9-18.

17. Kim S, Carlson K. Occurrence of ionophore antibiotics in water and sediments of a mixedlandscape watershed. Water Res. 2006;40(13):2549-60.

18. Gebara ECE, Zardetto CGDC, Mayer MPA. Estudo in vitro da ação antimicrobiana de substâncias naturais sobre S. mutans e S. sobrinus. Rev Odontol Univ São Paulo. 1996;10(4):251-6.

19. Oliveira JHHL, Granato AC, Hirata DB, Hokka CO, Barboza M, Trsic M. Ácido clavulânico e cefamicina c: uma perspectiva da biossíntese, processos de isolamento e mecanismo de ação. Quim Nova. 2009;32(8):2142-50. http://dx.doi.org/10.1590/S0100-40422009000800028.

20. Egger SS, Drewe J, Schlienger RG. Potential drug-drug interactions in the medication of medical patients at hospital discharge. Eur J Clin Pharmacol. 2003;58(11):773-8. https://doi.org/10.1007/s00228-002-0557-z.

21. Secoli SR. Polifarmácia: interações e reações adversas no uso de medicamentos por idosos. Rev Bras Enferm. 2010;63(1):136-40. http://dx.doi.org/10.1590/S0034-71672010000100023.

22. Halkin H, Katzir I, Kurman I, Jan J, Malkin BB. Preventing drug interactions by online prescription screening in community pharmacies and medical practices. Clin Pharmacol Ther. 2001;69(4):260-5. https://doi.org/10.1067/mcp.2001.114228.

23. Moura CS, Ribeiro AQ, Magalhães SMS. Avaliação de interações medicamentosas potenciais em prescrições médicas do Hospital das Clínicas da Universidade Federal de Minas Gerais (Brasil). Lat Am J Pharm. 2007;26(4):596-601.

24. Jacomini LCL, Silva NA. Interações medicamentosas: contribuiç̧ão para o uso racional de imunossupressores sintéticos e biológicos. Rev Bras Reumatol. 2011;51(2):161-74. http://dx.doi.org/10.1590/S0482-50042011000200006. 
25. Criado PR, Criado RFJ, Maruta CW, Machado Filho CA. Histamina, receptores de histamina e anti-histamínicos: novos conceitos. An Bras Dermatol. 2010;85(2):195-210.

http://dx.doi.org/10.1590/S0365-05962010000200010.

26. Cadar A, Guimarães RE. Phycomicose dos seios paranasais. Rev Bras Otorrinolaringol. 1974;40(2):139-41.

\section{Minicurrículo}

Patrick Leonardo Nogueira da Silva | ORCiD: 0000-0003-2399-9526

Especialista em Enfermagem do Trabalho pela Faculdade de Guanambi - FG. Enfermeiro da Fundação Hospitalar do Município de Espinosa - FHUMESP. Professor da Universidade Estadual de Montes Claros UNIMONTES.

Larissa Santos Alves | ORCiD: 0000-0001-9799-1696

Farmacêutica pelas Faculdades Unidas do Norte de Minas - FUNORTE.

José Ronivon Fonseca | ORCiD: 0000-0002-4318-7114

Mestre em Cuidado Primário em Saúde pela Universidade Estadual de Montes Claros - UNIMONTES. Enfermeiro e servidor público da Prefeitura Municipal de Montes Claros. Professor do Departamento de Enfermagem das Faculdades Unidas do Norte de Minas - FUNORTE.

Luçandra Ramos Espírito Santo | ORCID: 0000-0003-0588-6149

Doutora em Ciências da Saúde pela Universidade Estadual de Montes Claros - UNIMONTES. Professora do Departamento de Farmácia das Faculdades Unidas do Norte de Minas - FUNORTE.

Maria Dolores Tiago Vaz | ORCiD: 0000-0002-0066-4435

Mestre em Gestão em Saúde Pública pela Universidade Federal de Juiz de Fora - UFJF. Professora do Departamento de Farmácia das Faculdades Unidas do Norte de Minas - FUNORTE. 